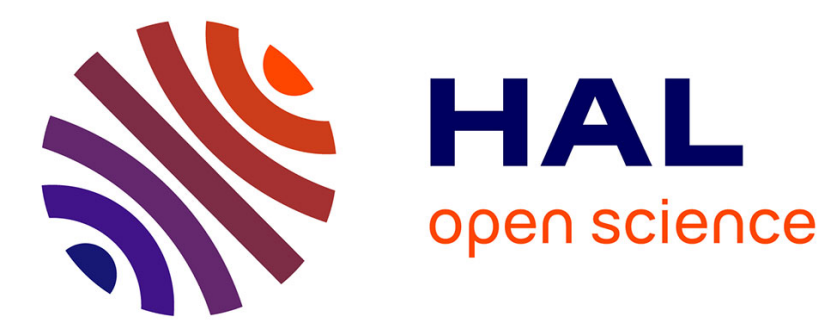

\title{
Connectedness and Local Search for Bicriteria Knapsack Problems
}

\author{
Arnaud Liefooghe, Luis Paquete, Marco Simoes, José Figueira
}

\section{To cite this version:}

Arnaud Liefooghe, Luis Paquete, Marco Simoes, José Figueira. Connectedness and Local Search for Bicriteria Knapsack Problems. 11th European Conference on Evolutionary Computation in Combinatorial Optimisation - EvoCOP 2011, Apr 2011, Torino, Italy. pp.48-59, 10.1007/978-3-642-203640_5 . hal-00575922

\section{HAL Id: hal-00575922 \\ https://hal.science/hal-00575922}

Submitted on 19 May 2011

HAL is a multi-disciplinary open access archive for the deposit and dissemination of scientific research documents, whether they are published or not. The documents may come from teaching and research institutions in France or abroad, or from public or private research centers.
L'archive ouverte pluridisciplinaire HAL, est destinée au dépôt et à la diffusion de documents scientifiques de niveau recherche, publiés ou non, émanant des établissements d'enseignement et de recherche français ou étrangers, des laboratoires publics ou privés. 


\title{
Connectedness and Local Search for Bicriteria Knapsack Problems
}

\author{
Arnaud Liefooghe ${ }^{1}$, Luís Paquete ${ }^{2}$, Marco Simões $^{2}$, and José R. Figueira ${ }^{3}$ \\ 1 Université Lille 1, LIFL - CNRS - INRIA Lille-Nord Europe, France \\ arnaud.liefooghe@univ-lille1.fr \\ 2 CISUC, Department of Informatics Engineering, University of Coimbra, Portugal \\ paquete@dei.uc.pt,msimoes@student.dei.uc.pt \\ 3 INPL, École des Mines de Nancy, Laboratoire LORIA, France \\ jose.figueira@mines.inpl-nancy.fr
}

\begin{abstract}
This article reports an experimental study on a given structural property of connectedness of optimal solutions for two variants of the bicriteria knapsack problem. A local search algorithm that explores this property is then proposed and its performance is compared against exact algorithms in terms of running time and number of optimal solutions found. The experimental results indicate that this simple local search algorithm is able to find a representative set of optimal solutions in most of the cases, and in much less time than exact approaches.
\end{abstract}

\section{Introduction}

Stochastic local search algorithms have been applied successfully to many multicriteria combinatorial optimization (MCO) problems. It is widely accepted that their sound performance is related to some structural properties of the solution space that allow local search procedures to find reasonably good quality solutions in an effective manner. However, little is known about which properties these are and how they can affect the performance of this class of algorithms.

In this article, the notion of connectedness of the set of optimal solutions for MCO problems (a.k.a. the efficient set) [3] is analyzed from an experimental point of view, and related to the performance of a particular class of multicriteria local search algorithms [9]. For a given efficient set of a MCO problem instance, a graph can be constructed such that each node represents an optimal solution and an edge connects two nodes if the corresponding optimal solutions are neighbors for a given neighborhood structure. The efficient set is connected with respect to that neighborhood structure if the underlying graph is also connected, that is, there is a path between any pair of nodes. If the efficient set is connected and the neighborhood structure is tractable from a computational point of view, local search algorithms would be able to find the efficient set in a very effective manner [9], by starting from at least one efficient solution. However, worst-case results have shown that the efficient set for many MCO problems is not connected in general with respect to different neighborhood structures $[3,5]$, except for very few particular cases $[6,11]$. Moreover, some recent results indicate that 
approximate solutions that are obtained from independent metaheuristic runs on the bicriteria traveling salesman problem are strongly clustered with respect to small-sized neighborhood structures [10].

The degree of connectedness is here investigated experimentally for two variants of the bicriteria knapsack problem: The bicriteria unconstrained optimization problem (BUOP) and the bicriteria knapsack problem with bounded cardinality (BKP-BC). Both problems are NP-hard and intractable in the general case [2]. The experimental results suggest that the efficient set for the two problems above is very often connected with respect to elementary neighborhood structures, despite of the negative results reported in the literature [5]. Based on these positive findings, a local search algorithm is proposed and its performance is compared with that of multicriteria dynamic programming algorithms in terms of running-time and number of optimal solutions found. A special technique is introduced that allows the early termination of the complete neighborhood exploration without harming algorithmic performance in terms of solution quality.

The article is organized as follows. Section 2 and Section 3 give the connectedness results for BUOP and BKP-BC, respectively. Moreover, for each of the sections above, the local search algorithms and the exact approaches for the corresponding problem are introduced. Numerical results are shown on a large set of random instances of different structure and size. Finally, Section 4 presents conclusions and further work.

\section{The Bicriteria Unconstrained Optimization Problem}

This section introduces a variant of the classical knapsack problem where the capacity constraint is transformed into an additional criterion to be optimized. Then, an experimental analysis on the connectedness property of the corresponding efficient set is reported, as well as the performance of a local search algorithm on several instances of the problem.

\subsection{Problem Definition}

The original (single-criterion) 0/1 knapsack problem is formulated as follows:

$$
\begin{aligned}
\max & \sum_{i=1}^{n} p_{i} x_{i} \\
\text { s.t. } & \sum_{i=1}^{n} w_{i} x_{i} \leq W
\end{aligned}
$$

where $p=\left(p_{1}, p_{2}, \ldots, p_{j}, \ldots, p_{n}\right)$ is the profit vector, $p_{j}$ representing the amount of profit on item $j, j=1, \ldots, n$, and $x=\left(x_{1}, x_{2}, \ldots, x_{j}, \ldots, x_{n}\right)$ with $x_{j}=1$ if the item $j$ is included in the subset of selected items (knapsack) and $x_{j}=0$ otherwise; $w=\left(w_{1}, w_{2}, \ldots, w_{j}, \ldots, w_{n}\right)$ is the weight vector, $w_{j}$ representing the amount of investment on item $j, j=1, \ldots, n$; and $W$ is the overall amount 
available or budget. The sum of profits and the sum of weights of a given solution $x$ are denoted by $p(x)$ and $w(x)$, respectively.

By transforming the capacity constraint of Problem (1) into a criterion, the following bicriteria unconstrained optimization problem [2] is obtained:

$$
\max (p(x),-w(x)) .
$$

A proper meaning to the operator "max" above is given as follows. Let $X$ denote the set of feasible solutions of Problem (2). The image of the feasible solutions when using the vector maximizing function of Problem (2) defines the feasible region in the criteria space, denoted here by $Z \subseteq \mathbb{N}^{2}$. A feasible solution $x \in X$ is efficient if there does not exist another feasible solution $x^{\prime} \in X$ such that $p\left(x^{\prime}\right) \geq p(x)$ and $w\left(x^{\prime}\right) \leq w(x)$, with at least one strict inequality in one of above (or $\left.\left(p\left(x^{\prime}\right),-w\left(x^{\prime}\right)\right) \geq(p(x),-w(x))\right)$. A vector $z \in Z$ is nondominated if there is some efficient solution $x$ such that $z=(p(x),-w(x))$. A vector $z \in Z$ dominates a vector $z^{\prime} \in Z$ (or $z^{\prime}$ is dominated by $z$ ) if $z \geq z^{\prime}$ holds; if neither $z \nsupseteq z^{\prime}$ nor $z^{\prime} \nsucceq z$ holds, then both are (mutually) nondominated. The set of all efficient solutions and the set of nondominated vectors are called the efficient set and the nondominated set, respectively. The usual goal of MCO is to find a minimal complete set, that is, the smallest subset of the efficient set whose image coincides with the nondominated set. This subset may not be unique.

\subsection{Connectedness Analysis}

This section describes an experimental analysis for investigating the influence of problem size and degree of conflict between the two criteria on the connectedness property of the efficient set for BUOP. A multicriteria dynamic programming (MDP-BUOP) algorithm is implemented to compute the efficient set. This algorithm consists of the first phase of the Nemhauser-Ullman algorithm for the single-criterion $0 / 1$ knapsack problem [8]. It has shown to be theoretically efficient for several input data distributions [1]. The MDP-BUOP sequential process consists of $n$ stages. At any stage $i$, the algorithm generates a set $S_{i}$ of states, which represents a set of promising feasible solutions made up of the first $i$ items, $i=1, \ldots, n$. A state $s=\left(s^{p}, s^{w}\right) \in S_{i}$ represents a feasible solution of profits $s^{p}$ and weight $s^{w}$. The MDP-BUOP algorithm follows the recursion:

$$
S_{i}:=\operatorname{vmax}\left\{\left(s^{p}+p_{i}, s^{w}-w_{i}\right), s \in S_{i-1}\right\}
$$

for $i=1, \ldots, n$, with the basis case $S_{0}:=(0,0)$. Operator "vmax" returns the states that are nondominated in $S_{i}$. At the last stage $n$, the set $S_{n}$ corresponds to the nondominated set.

In order to obtain the efficient set with the MDP-BUOP algorithm, a binary string is generated with each new state and updated accordingly during the sequential process. For this reason, the implementation keeps states with the same component values. The removal of dominated states at each stage is performed by the algorithm of Kung et al. [7]. Only two sets of states are maintained during the overall sequential process since, at any stage $i>0$, only set $S_{i-1}$ is required. 
Table 1. Percentage of BUOP instances connected with respect to the 1-flip neighborhood (all instances are connected with respect to the 1-flip-exchange neighborhood) and average size of the efficient set for each instance size and data correlation (\%con and avgs, respectively).

\begin{tabular}{r|rr|rc|rc|rr|rr} 
& \multicolumn{2}{|c|}{$\rho=-0.8$} & \multicolumn{2}{c|}{$\rho=-0.4$} & \multicolumn{2}{c|}{$\rho=0.0$} & \multicolumn{2}{c|}{$\rho=0.4$} & \multicolumn{2}{c}{$\rho=0.8$} \\
size & \%con & \multicolumn{1}{c|}{ avgs } & \%con & \multicolumn{1}{c}{ avgs } & \%con & avgs & \%con & avgs & \%con & \multicolumn{1}{c}{ avgs } \\
\hline 300 & 100.0 & 8965 & 96.7 & 10838 & 100.0 & 14501 & 100.0 & 24702 & 93.3 & 55159 \\
600 & 96.7 & 32242 & 100.0 & 39056 & 100.0 & 52675 & 96.7 & 92717 & 90.0 & 228427 \\
900 & 90.0 & 69208 & 93.3 & 83357 & 86.7 & 112598 & 76.7 & 201642 & 60.0 & 495154 \\
1200 & 96.7 & 118483 & 90.0 & 144259 & 86.7 & 195396 & 83.3 & 338903 & - & 823612 \\
1500 & 90.0 & 179932 & 93.3 & 217230 & 86.7 & 301845 & - & 526029 & - & 1352817 \\
1800 & 83.3 & 252972 & 93.3 & 308373 & - & 423450 & - & 716598 & - & 1818608 \\
2100 & 76.7 & 337443 & - & 409771 & - & 563840 & - & 969069 & - & 2431715 \\
\hline
\end{tabular}

These two sets are implemented as height-balanced binary search trees in order to allow logarithmic-time operations. If the implementation does not terminate before one hour of CPU-time, or if RAM resources available are exceeded, the run is cancelled and the output is omitted. The code is written in $\mathrm{C}++$.

BUOP instances are defined with two parameters: problem size $(n)$ and correlation between profit and weight vectors $(\rho)$. Both parameters affect the size of the efficient set. The positive (resp. negative) data correlation will increase (resp. decrease) the degree of conflict between the two criteria. The size of the instances ranges from 300 to 3000 and the correlations are $\rho \in\{-0.8,-0.4,0.0,0.4,0.8\}$. Profit and weight integer values are generated randomly according to a uniform distribution in $[1, M / n]$, where $M$ denotes the maximum possible integer value. The generation of correlated data follows the procedure given by Verel et al. [12] For each problem size and each correlation degree, 30 different and independent instances are randomly generated.

Two neighborhood structures are considered for the experimental analysis: the 1-flip and 1-flip-exchange neighborhoods. Two feasible solutions are 1flip neighbors if they differ exactly on one assignment. In other words, a given neighbor can be reached by adding or removing one item from a given solution. Hence, this neighborhood structure is directly related to the Hamming distance between binary strings. The 1-flip-exchange neighborhood is an extension of the neighborhood above. Two feasible solutions are 1-flip-exchange neighbors if one can be obtained from the other by exchanging two items, adding one item, or removing one item. The size of the neighborhood is linear with $n$ for the 1flip neighborhood structure, while it is quadratic in the case of 1-flip-exchange. Both neighborhoods coincide with the neighborhood structures used by Gorski et al. [5] for a similar class of problems.

For the connectedness analysis, MDP-BUOP outputs the efficient set for every instance. For each neighborhood structure, an adjacency matrix is built, indicating whether each two efficient solutions are neighbors or not. Based on this matrix, the connectedness of the corresponding graph is tested. Since this 


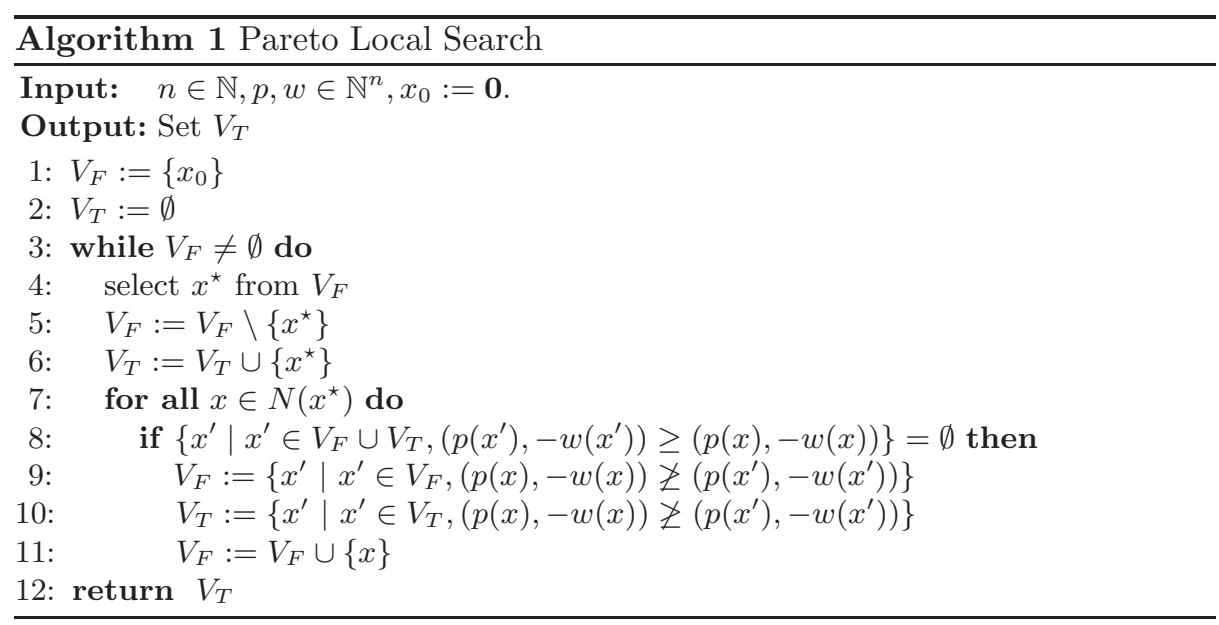

analysis involves a large usage of memory resources (more than $2 \mathrm{~Gb}$ for largesize instances), only results for a limited number of instance sizes and correlation values are presented. Table 1 gives the percentage of instances with the efficient set that is connected with respect to the 1-flip neighborhood, as well as the average size of the efficient set, rounded to the nearest integer. Although the correlation in the input data influences the size of the efficient set, it does not seem to affect the connectedness results. However, the proportion of instances with a connected efficient set slightly decreases with the increase of the instance size. Finally, for those set of instances, the efficient set is always connected with respect to the 1-flip-exchange neighborhood.

\subsection{Local Search for BUOP}

The local search algorithm for BUOP (PLS-BUOP) is based on the Pareto Local Search [9]. The pseudo-code is given in Algorithm 1. For simplification purpose, it is assumed that all feasible solutions have a distinct image in the criterion space. Two archives of nondominated solutions are maintained, $V_{T}$ and $V_{F}$, respectively. Archive $V_{T}$ contains the set of solutions whose neighborhood has already been explored, while $V_{F}$ contains the remaining ones. PLS-BUOP starts with an efficient solution that initializes the archive. Then, at each iteration, a solution is chosen from $V_{F}$, and its neighborhood is explored $(\mathcal{N}(x)$ denotes the neighborhood of a given solution $x \in X$ ). All the nondominated neighboring solutions are used to update $V_{F}$ and dominated solutions are discarded from $V_{F}$ and $V_{T}$. The algorithm terminates once $V_{F}$ is empty. This algorithm stops naturally when a Pareto local optimum set is found, it does not cycle, and if connectedness of the efficient set holds, it is able to identify it by starting from at least one efficient solution (with a proper change of the conditions in Algorithm 1) [9]. In the following, some particular details of the implementation are described. 

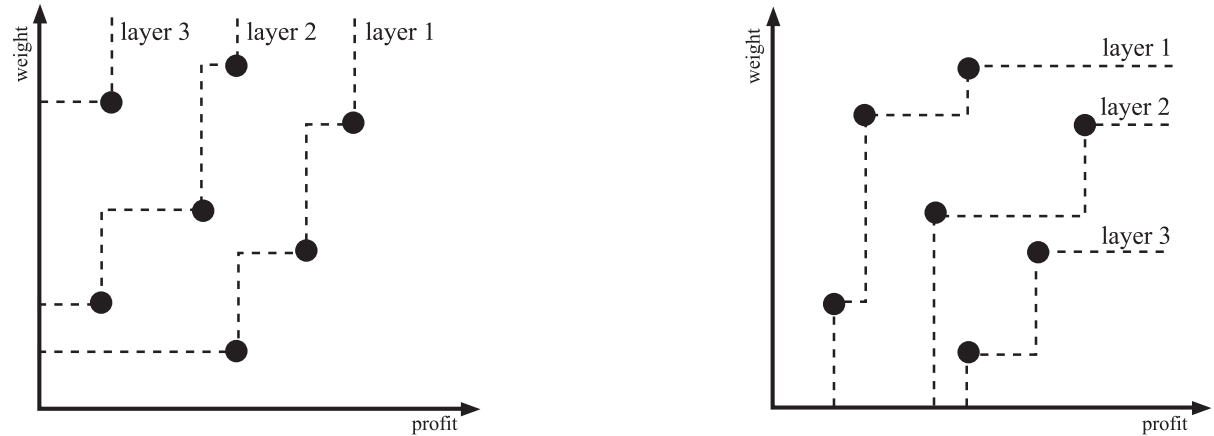

Fig. 1. Initial dominance-depth ranking of items for the exploration of the neighborhood (left: ranking for adding, right: ranking for deleting).

- Initialization. The algorithm starts with the efficient solution $x_{0}=\mathbf{0}$. This solution maps, in the criteria space, to an extreme point from the nondominated set, with null profit and weight values.

- Selection. At each iteration of the algorithm, the solution from $V_{F}$ with the smallest profit value is selected (Algorithm 1, line 4).

- 1-flip neighborhood exploration. In order to perform the 1-flip neighborhood exploration in an efficient manner, a preliminary step ranks the items into different layers, with respect to the dominance-depth ranking [4]. Two different ranks are required to cover the cases of adding and deleting an item. This step is illustrated in Fig. 1. Let $L_{i}$ denote the set of items in the $i$-th layer and let $u \in L_{i}$ and $v \in L_{j}, j>i$. Then, for the case of the addition, it holds that $\left(p_{v},-w_{v}\right) \nsupseteq\left(p_{u},-w_{u}\right)$. Therefore, the neighborhood exploration starts by examining the items in the first layer, and proceeds with the items in the subsequent layers. Within the same layer, the exploration follows the nondecreasing order of the weights. The exploration stops after verifying that no item of a given layer belongs to the current solution. Indeed, any neighboring solution constructed from the subsequent layers is dominated by at least one neighboring solution built from this layer. For the case of deletion, a similar reasoning applies with the corresponding changes.

- 1-flip-exchange neighborhood exploration. For this neighborhood, the same reasoning as above applies when adding or removing an item. In order to exchange pairs of items more efficiently, the following pre-processing procedure is applied: First, for each item $i \in\{1, \ldots, n\}$, a tuple records the profit and weight difference with respect to each different item $j$. Then, the $n-1$ tuples are sorted in terms of dominance-depth ranking. When considering the exchange of item $i$ with another item, the exploration follows the order given by the dominance-depth ranking, with the nondecreasing order of the weighs for tuples within the same layer; the exploration stops once the items corresponding to all tuples of a given layer can be exchanged with $i$. The exploration is iterated for every item in the knapsack. 

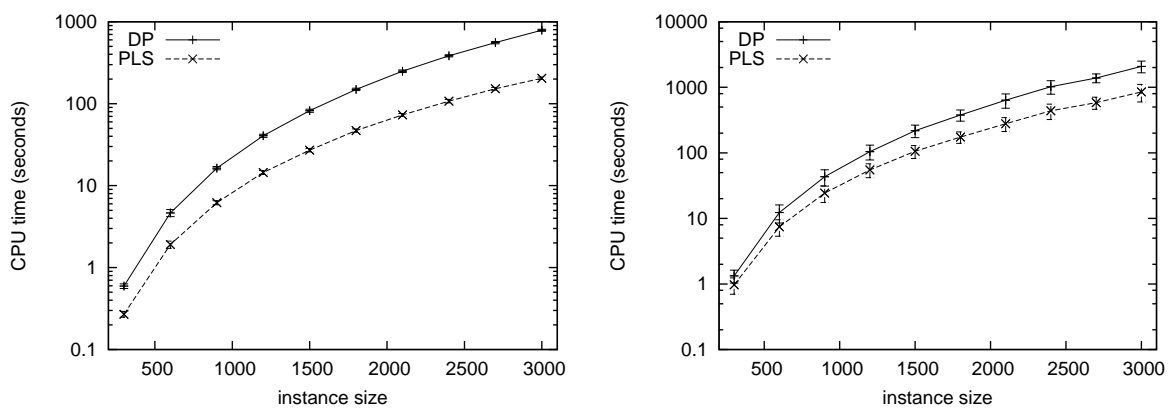

Fig. 2. CPU time in seconds (average value and standard deviation, given in log scale) of MDP-BUOP and PLS-BUOP using the 1-flip neighborhood for instances with data correlation $\rho=-0.4$ (left) and $\rho=0.4$ (right).

- Data structures. Archives $V_{F}$ and $V_{T}$ are implemented as height-balanced binary search trees. The removal of dominated solutions follows the algorithm of Kung et al. [7].

MDP-BUOP and two PLS-BUOP versions, each one for each neighborhood structure, were run on the instances described in Section 2.2. The implementation of MDP-BUOP was modified in order to output a minimal complete set. All the algorithms share the same programming language, data structures, compilation options and were run in the same machine with a time bound of one hour. The PC used for the experiments was an iMac with Mac OS X v10.5.5 (Leopard), $2.4 \mathrm{GHz}$ Intel Core with 4MB L2 Cache and 2GB SDRAM. All codes were compiled with $\mathrm{g}++$ version 4.0.1 using the -O3 flag. The CPU-time taken by MDP-BUOP and PLS-BUOP with 1-flip neighborhood for instances with correlation $\rho=-0.4$ and $\rho=0.4$ is reported in Fig. 2. In order to distinguish the differences of performance, the CPU-time is presented in log scale. Similar relationship between performance of both implementations was obtained for the remaining correlation values. Table 2 presents the percentage of efficient solutions and the total number of solutions returned by PLS-BUOP using 1flip neighborhood, averaged over results obtained in 30 instances for each size and correlation. Since PLS-BUOP using the 1-flip-exchange neighborhood took always more time than MDP-BUOP, the results of the former are omitted.

Although the experimental analysis indicated the existence of instances with unconnected efficient set with respect to the 1-flip neighborhood (see Table 1), the results show that the local search approach using the same notion of neighborhood is able to identify a minimal complete set in many cases. For other instances, it leads to the identification of more than $99.9 \%$ of the efficient set. Furthermore, the local search performs very efficiently in comparison to the dynamic programming approach in terms of computational time. Indeed, the larger the instance size, the larger the gap between PLS-BUOP and MDP-BUOP in 
Table 2. Average percentage of efficient solutions and average number of solutions found by PLS-BUOP using the 1-flip neighborhood (\%ef and avgs, respectively).

\begin{tabular}{|c|c|c|c|c|c|c|c|c|c|c|}
\hline \multirow[b]{2}{*}{ size } & \multicolumn{2}{|c|}{$\rho=-0.8$} & \multicolumn{2}{|c|}{$\rho=-0.4$} & \multicolumn{2}{|c|}{$\rho=0.0$} & \multicolumn{2}{|c|}{$\rho=0.4$} & \multicolumn{2}{|c|}{$\rho=0.8$} \\
\hline & $\% e f$ & avgs & $\% e f$ & avgs & $\% e f$ & avgs & $\% e f$ & avgs & $\% e f$ & avgs \\
\hline 300 & 100.0 & 8965 & 99.9 & 10838 & 100.0 & 14501.2 & 100.0 & 24702 & 99.9 & 55159 \\
\hline 600 & 99.9 & 32242 & 100.0 & 39056 & 100.0 & 52675.4 & 100.0 & 92717 & 100.0 & 228427 \\
\hline 900 & 99.9 & 69208 & 99.9 & 83357 & 99.9 & 112597.3 & 99.9 & 201642 & 99.9 & 495153 \\
\hline 1200 & 100.0 & 118483 & 99.9 & 144259 & 99.9 & 195395.6 & 100.0 & 338903 & 99.9 & 823611 \\
\hline 1500 & 99.9 & 179932 & 100.0 & 217230 & 100.0 & 301844.6 & 99.9 & 526029 & 99.9 & 1352816 \\
\hline 1800 & 99.9 & 252972 & 100.0 & 308373 & 99.9 & 423450.0 & 99.9 & 716598 & 99.9 & 1818607 \\
\hline 2100 & 99.9 & 337443 & 99.9 & 409770 & 99.9 & 563839.5 & 99.9 & 969068 & 99.9 & 2431713 \\
\hline 2400 & 100.0 & 434333 & 99.9 & 530843 & 100.0 & 719388.3 & 99.9 & 1300909 & - & 3358473 \\
\hline 2700 & 99.9 & 541046 & 100.0 & 661676 & 100.0 & 900284.3 & 100.0 & 1560123 & - & 4158499 \\
\hline 3000 & 99.9 & 658680 & 100.0 & 806236 & 100.0 & 1100882.2 & 99.9 & 2022463 & - & 4846109 \\
\hline
\end{tabular}

terms of CPU time. However, PLS-BUOP appears to be much more efficient for negatively correlated data, while MDP-BUOP was not able to solve all the instances for positively correlated profit and weight values.

\section{The Bicriteria Knapsack Problem with Bounded Cardinality}

This section reports a similar analysis on a variant of the previous problem, where an additional cardinality constraint is considered.

\subsection{Problem Definition}

The bicriteria knapsack problem with bounded cardinality is a variant of the BUOP that is obtained by limiting the number of chosen items by a cardinality bound $(k)$. The formulation of BKP-BC is as follows:

$$
\begin{aligned}
& \max (p(x),-w(x)) \\
& \text { s.t. } \sum_{i=1}^{n} x_{i} \leq k
\end{aligned}
$$

In the problem above, the operator "max" follows the same meaning as in Problem (2). The same terminology and notation will be used for this problem.

\subsection{Connectedness Analysis}

The efficient set is computed by means of a multicriteria dynamic programming (MDP-BKP-BC) algorithm that extends the MDP-BUOP algorithm given in Section 2.2. This algorithm has $k \times n$ stages. At each stage, the algorithm 
generates the set $T_{(i, j)}$ of states, which represents a set of potential efficient solutions made up of the first $i$ items, $i=1, \ldots, n$, with cardinality $j, j=1, \ldots, k$. A state $t=\left(t^{p}, t^{w}\right) \in T_{(i, j)}$ represents a feasible solution of profits $t^{p}$ and weight $t^{w}$. This approach follows the recurrence relation:

$$
T_{(i, j)}:=\operatorname{vmax}\left(T_{(i-1, j)} \cup\left\{\left(t^{s}+p_{i}, t^{w}-w_{i}\right), t \in T_{(i-1, j-1)}\right\}\right)
$$

for $i=1, \ldots, n$ and $j=1, \ldots, k$, with the basis cases $T_{(i, 0)}:=(0,0)$, for $i=$ $0, \ldots, n$ and $T_{(0, j)}:=(0,0)$ for $j=0, \ldots, k$. The nondominated set of states is given by the set $\operatorname{vmax}\left(T_{(n, 0)} \cup \cdots \cup T_{(n, k)}\right)$. The implementation follows the same principles presented in Section 2.2 for the MDP-BUOP algorithm. However, the implementation of MDP-BKP-BC has to keep $2(k+1)$ sets during the overall process, since at each state $i$, the sets $T_{(i, j)}$ and $T_{(i-1, j)}$, for $j=0, \ldots, k$ are required. For this reason, MDP-BKP-BC should take more time than MDPBUOP for the same instance size.

A similar set of instances to those used for the previous problem is generated. In addition to the instance size $(n)$ and to the data correlation $(\rho)$, several different values for the cardinality bound $(k)$ are considered: $n / 10, n / 5$, and $n / 2$. For each problem size, correlation and cardinality bound, 30 different and independent instances are generated randomly, as explained in Section 2.2. The two neighborhood structures described in Section 2.2 were also considered for this problem. The use of the 1-flip-exchange neighborhood for this problem is motivated by the conjecture that many efficient solutions may have the same cardinality, due to the additional cardinality constraint.

Due to limited memory resources, results were only obtained for instances of limited size (these instances can be inferred from Table 3). Differently from the connectedness results obtained in the first problem (see Table 1), no instance with an efficient set that is connected with respect to the 1-flip neighborhood was found. However, the efficient set for all the instances were connected with respect to the 1-flip-exchange neighborhood. These results corroborate those of Gorski et al. [5] for much smaller instances (up to 100 items).

\subsection{Local Search for BKP-BC}

Given the positive results reported in the previous section, a local search (PLSBKP-BC) was developed under the same reasoning of PLS-BUOP (see Section 2.3). The only difference is in the neighborhood exploration since a maximum number of items has to be ensured in the solution when considering the possibility of adding an item.

MDP-BKP-BC and two versions of PLS-BKP-BC were run in the same instances defined in the previous section. The experiments were performed in a computer cluster with 6 nodes, each with an AMD Phenom II X6 processor with $3.2 \mathrm{GHz}, 3$ and $6 \mathrm{MB}$ L2 and L3 Cache, respectively, and $12 \mathrm{MB}$ DDR3 SDRAM. The operating system was Ubuntu 8.04 LTS. Both codes were compiled with gcc version 4.2.4 using the -O3 flag.

The CPU-time taken by the three approaches is plotted in Fig. 3 for four different instance parameter settings. In order to distinguish the differences of 

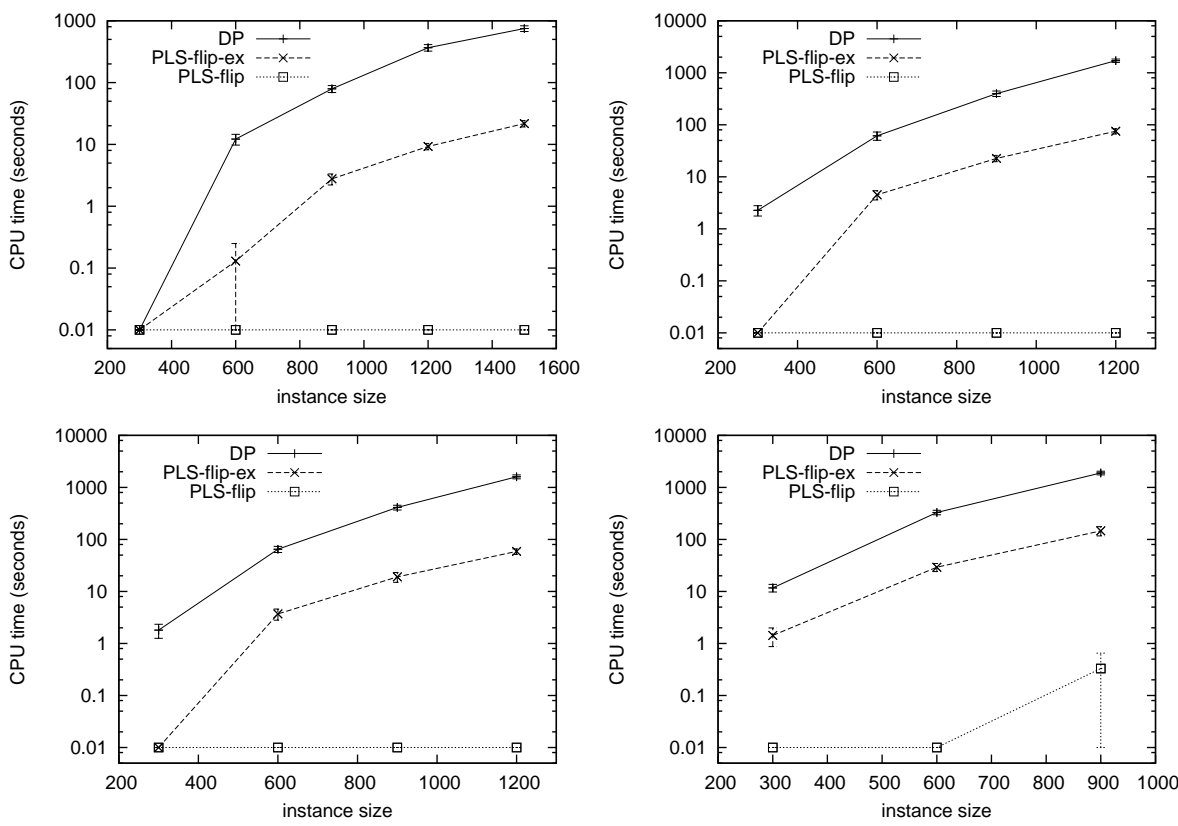

Fig. 3. CPU time in seconds (average value and standard deviation, given in log scale) of the three approaches for instances with parameters $k=n / 10$ (top) and $k=n / 5$ (bottom), $\rho=-0.4$ (left) and $\rho=0.4$ (right).

performance, the CPU-time is presented in log scale. Clearly, both PLS-BKP$\mathrm{BC}$ versions take much less time than MDP-BKP-BC to terminate. Similar results hold for the remaining instances. Table 3 reports the percentage of efficient solutions found by the two versions of PLS-BKP-BC. As expected from the connectedness analysis reported in the previous section, PLS-BKP-BC using the 1-flip-exchange neighborhood was always able to find a minimal complete set for all instances up to 1800 items in less than one hour of CPU-time. PLS-BKP$\mathrm{BC}$ using 1-flip neighborhood is, in many cases, able to find more than half of a minimal complete set in less than one second.

\section{Concluding Remarks}

This article describes an experimental analysis on the structure of the efficient set, in terms of connectedness, for two MCO problems. Despite of the negative results reported in the literature for similar problems [3,5], the experimental analysis for the problems investigated in this paper are quite promising. For both bicriteria versions of the unconstrained optimization problem and knapsack problem with a bounded cardinality constraint, the experiments suggest that small-sized neighborhood structures give rise to connected efficient sets quite 
Table 3. Average percentage of efficient solutions found by PLS-BKP-BC using the 1-flip and the 1-flip-exchange neighborhood structures (\%e $f_{1}$ and $\% e f_{2}$, respectively).

\begin{tabular}{|c|c|c|c|c|c|c|c|c|c|c|c|}
\hline \multirow[b]{2}{*}{ size } & \multirow[b]{2}{*}{ card. } & \multicolumn{2}{|c|}{$\rho=-0.8$} & \multicolumn{2}{|c|}{$\rho=-0.4$} & \multicolumn{2}{|c|}{$\rho=0.0$} & \multicolumn{2}{|c|}{$\rho=0.4$} & \multicolumn{2}{|c|}{$\rho=0.8$} \\
\hline & & $\% e f_{1}$ & $\% e f_{2}$ & $\% e f_{1}$ & $\% e f_{2}$ & $\% e f_{1}$ & $\% e f_{2}$ & $\% e f_{1}$ & $\% e f_{2}$ & $\% e f_{1}$ & $\% e f_{2}$ \\
\hline \multirow[t]{3}{*}{300} & $n / 10$ & 72.6 & 100.0 & 54.1 & 100.0 & 42.0 & 100.0 & 44.2 & 100.0 & 47.7 & 100.0 \\
\hline & $n / 5$ & 83.9 & 100.0 & 63.0 & 100.0 & 53.5 & 100.0 & 54.4 & 100.0 & 56.3 & 100.0 \\
\hline & $n / 2$ & 95.6 & 100.0 & 88.6 & 100.0 & 85.3 & 100.0 & 83.1 & 100.0 & 84.7 & 100.0 \\
\hline \multirow[t]{3}{*}{600} & $n / 10$ & 75.3 & 100.0 & 50.9 & 100.0 & 41.6 & 100.0 & 41.6 & 100.0 & 47.6 & 100.0 \\
\hline & $n / 5$ & 84.2 & 100.0 & 63.3 & 100.0 & 51.3 & 100.0 & 50.0 & 100.0 & 55.3 & 100.0 \\
\hline & $n / 2$ & 95.4 & 100.0 & 88.3 & 100.0 & 83.1 & 100.0 & 83.4 & 100.0 & - & - \\
\hline \multirow[t]{2}{*}{900} & $n / 10$ & 75.6 & 100 & 50.1 & 10 & 39.6 & 100.0 & 40.5 & 100.0 & 47.0 & 100.0 \\
\hline & $n / 5$ & 83.1 & 100 & 0 & 10 & 50.4 & 10 & 51.5 & & - & - \\
\hline \multirow[t]{2}{*}{1200} & $n / 10$ & 75.6 & 100 & 50 & 100 & 40.2 & 100.0 & 39.9 & 100.0 & - & - \\
\hline & $n / 5$ & 82.3 & 100.0 & 60.0 & 100.0 & 50.9 & 100.0 & - & - & - & - \\
\hline \multirow[t]{2}{*}{1500} & $n / 10$ & 75.4 & 100.0 & 50.5 & 100.0 & 40.3 & 100.0 & - & - & - & - \\
\hline & $n / 5$ & 83.1 & 100.0 & - & - & - & - & - & - & - & - \\
\hline 1800 & $n / 10$ & 75.4 & 100.0 & - & - & - & - & - & - & - & - \\
\hline
\end{tabular}

frequently, and independently of the size and of the structure of input data. In fact, it is not yet clear what structure of the input data may generate, in general, an unconnected efficient set under the 1-flip-exchange neighborhood that was used in this article.

Although the large number of connected instances motivates the use of local search algorithms, it is still an open question whether those approaches are efficient enough as compared to exact algorithms. The experimental analysis reported in this article gives a clear positive answer for the second problem. For the first problem, without cardinality constraint, some preliminary results indicated that the local search proposed in this article under the same neighborhood that (empirically) provides connectedness would not be worthwhile in terms of running time. Still, using a smaller neighborhood structure allows the same algorithm to find more than $99.9 \%$ of the efficient set in a significantly less amount of time than the exact approach.

The simplicity of the local search approach proposed in this article is very appealing for implementation purpose, needs no definition of parameters and requires a minimum number of modifications in order to be applied to other type of knapsack problems. For instance, the same principles can be applied to the multicriteria knapsack problem (with several maximizing profit criteria and one capacity constraint) by ignoring (or penalizing) infeasible neighboring solutions. However, finding appropriate definitions of neighborhoods that give rise to a large number of connected efficient sets for knapsack problems with capacity constraints is still under investigation.

A natural question is whether it is possible to derive analytical results for MCO problems that would prove connectedness by assuming some structure or distribution on the input data. Connections with neighborhood structures arising 
in the context of the linear programming formulation of the MCO problem may provide further insights [5]. Moreover, the derivation of bounds on the run-time of multiobjective evolutionary algorithms that start from efficient solutions are also of interest. For instance, the size of the efficient set for the first problem is polynomially bounded for many input data distributions [1]. This suggests that a polynomial run-time bound may be achieved by such type of algorithms.

Acknowledgements. The authors acknowledge Jochen Gorski for the discussion on the main topic of this article. This work was partially supported by the Portuguese Foundation for Science and Technology (PTDC/EIA-CCO/098674/2008) and the project "Connectedness and Local Search for Multiobjective Combinatorial Optimization" funded by the Deutscher Akademischer Austausch Dienst and Conselho de Reitores das Universidades Portuguesas. The third author acknowledges a CEG-IST grant from Instituto Superior Técnico (PTDC/GES/73853/2006).

\section{References}

1. Beier, R., Vöcking, B.: Probabilistic analysis of knapsack core algorithms. In: Proc. of the 15th ACM-SIAM Symposium on Discrete Algorithms (SODA 2004). pp. 468-477 (2004)

2. Ehrgott, M.: Multicriteria optimization, Lecture Notes in Economics and Mathematical Systems, vol. 491. Springer (2000)

3. Ehrgott, M., Klamroth, K.: Connectedness of efficient solutions in multiple criteria combinatorial optimization. European Journal of Operational Research 97(1), 159166 (1997)

4. Goldberg, D.: Genetic Algorithms in Search, Optimization and Machine Learning. Addison-Wesley, Boston, MA, USA (1989)

5. Gorski, J., Klamroth, K., Ruzika, S.: Connectedness of efficient solutions in multiple objective combinatorial optimization. Tech. Rep. 102/2006, University of Kaiserslautern, Department of Mathematics (2006)

6. Gorski, J., Paquete, L.: On a particular case of the multi-criteria unconstrained optimization problem. Electronic Notes on Discrete Mathematics 36, 135-142 (2010)

7. Kung, H., Luccio, F., Preparata, F.: On finding the maxima of a set of vectors. Journal of ACM 22(4), 469-476 (1975)

8. Nemhauser, G., Ullman, Z.: Discrete dynamic programming and capital allocation. Management Science 15(9), 494-505 (1969)

9. Paquete, L., Schiavinotto, T., Stützle, T.: On local optima in multiobjective combinatorial optimization problems. Annals of Operations Research 156(1), 83-97 (2007)

10. Paquete, L., Stützle, T.: Clusters of non-dominated solutions in multiobjective combinatorial optimization: An experimental analysis. In: Multiobjective Programming and Goal Programming, Lecture Notes in Economics and Mathematical Systems, vol. 618, pp. 69-77. Springer (2009)

11. da Silva, C.G., Clímaco, J., Figueira, J.R.: Geometrical configuration of the Pareto fronteir of the bi-criteria 0-1-knapsack problem. Tech. Rep. 16/2004, INESC, Coimbra, Portugal (2004)

12. Verel, S., Liefooghe, A., Jourdan, L., Dhaenens, C.: Analyzing the effect of objective correlation on the efficient set of MNK-landscapes. In: Proc. of the 5th Conference on Learning and Intelligent OptimizatioN (LION 5). Lecture Notes in Computer Science, Springer (2011), to appear 